\title{
Disnea de causa insospechada
}

\author{
Melissa Rodríguez Israel* \\ Andrés Garzona Navas** \\ Ana Beatriz Argüelles Argüello** \\ Ruy Vargas Baldares*** \\ * Servicio de Cardiología. Hospital San Vicente Paúl. Heredia. Costa Rica \\ * Servicio de Cardiología. Hospital México. San José. Costa Rica \\ ** Servicio de Anatomía Patológica. Hospital San Vicente Paúl. Heredia. Costa Rica
}

Recibido: 02/03/2020

Aceptado: 29/05/2020

En línea: 31/07/2020

Citar como: Rodríguez-Israel M, Garzona-Navas A, Argüelles-Argüello AB, Vargas-Baldares R. Disnea de causa insospechada. Rev Ecocar Pract (RETIC). 2020 (Jul); 3 (2): 18-21. doi: 10.37615/retic.v3n2a6.

Cite this as: Rodríguez-Israel M, Garzona-Navas A, Argüelles-Argüello AB, Vargas-Baldares R. Dyspnea ocurring from an unsuspected site. Rev Ecocar Pract (RETIC). 2020 (Jul); 3 (2): 18-21. doi: 10.37615/retic.v3n2a6.

\section{Palabras clave \\ $\triangleright$ Angiosarcoma \\ $\triangleright$ Tumor pericárdico \\ $\triangleright$ Hemopericardio}

\begin{tabular}{l}
\hline Keywords \\
\hline$\triangleright$ Angiosarcoma \\
$\triangleright$ Pericardial tumor \\
$\triangleright$ Hemopericardium \\
\end{tabular}

\section{RESUMEN}

El angiosarcoma primario de pericardio es una patología extremadamente infrecuente, con muy mal pronóstico. Se presenta un paciente ingresado por un cuadro constitucional y disnea en el que se diagnostica un derrame pericárdico hemorrágico asociado a un tumor maligno de pericardio, y que termina falleciendo. La autopsia confirmó el diagnóstico de angiosarcoma de pericardio.

ABSTRACT
Primary pericardial angiosarcoma is an extremely rare tumor associated with a very poor prognosis. This is a pre-
sentation of a case of a patient presenting with acute constitutional symptoms and dyspnea in whom a hemope-
ricardium due to a pericardial tumor. The case evolved rapidly and ended fatally. The diagnosis of primary pericar-
dial angiosarcoma was made in the autopsy.

\section{Presentación del caso}

Varón de 44 años, sin antecedentes patológicos relevantes que acudió al Servicio de Urgencias por un cuadro de astenia, adinamia, disnea y sensación febril. En la valoración inicial se apreció febrícula de $37,5^{\circ} \mathrm{C}$, derrame pleural bilateral moderado (Figura 1) y derrame pericárdico severo con signos de taponamiento cardíaco y compromiso hemodinámico, por lo que se procedió a drenar el derrame pericárdico, obteniendo $400 \mathrm{ml}$ de líquido sanguinolento.

El paciente ingresó en la Unidad de Cuidados Intensivos, donde se mantuvo en observación. El líquido pericárdico resultó ser un exudado, con resultados negativos en la inmunología para tuberculosis, las serologías virales y el estudio bacteriológico. Su condición clínica mejoró, por lo que se decidió darle el alta médica tras una ecocardiografía transtorácica (ETT) de control informada como normal (Vídeo 1).

Un mes después el paciente reingresa con mayor disnea, dolor torácico que empeora con el esfuerzo y fiebre persistente. Al examen físico se observa deterioro del estado general, disminución significativa de peso, fiebre y gesto facial de dolor al inspirar.
La radiografía de tórax muestra un parénquima pulmonar con infiltrados algodonosos difusos bilaterales y derrame pleural bilateral de predominio izquierdo (Figura 2). Se realiza una ETT en la que se observa un engrosamiento muy llamativo de hasta $5 \mathrm{~cm}$ del pericardio con una textura heterogénea en empedrado. Además, existía un movimiento disincrónico del septum interventricular muy llamativo (Vídeo 2, Vídeo 3 y Vídeo 4). En la tomografía de tórax se comprueba que el pericardio está muy engrosado, con múltiples calcificaciones nodulares de hasta $28 \mathrm{~mm}$ de diámetro. Además, se observa infiltrado alveolar, derrame pleural y atelectasia del lóbulo pulmonar inferior izquierdo (Figura 3). Se realizó una biopsia pleural sin resultado concluyente.

El estado clínico del paciente se deterioró rápidamente, por lo que no se pudo realizar una biopsia del pericardio y los cirujanos cardiovasculares rechazaron la posibilidad de realizar un abordaje quirúrgico. Evolucionó a shock cardiogénico con posterior claudicación ventilatoria. Se realizó una nueva una pericardiocentesis, obteniendo $600 \mathrm{ml}$ de líquido francamente hemorrágico. Horas después el paciente falleció. La autopsia evidenció un pericardio infiltrado por tejido necrótico y hemorrágico que formaba granulomas de gran tamaño (Figura 4 y Figura 5). El parénquima pulmonar también se encontraba invadido por lesiones nodulares (Figura 6). El análisis histológico dio el diagnóstico final de angiosarcoma primario de pericardio. 


\section{Estudio por imagen}

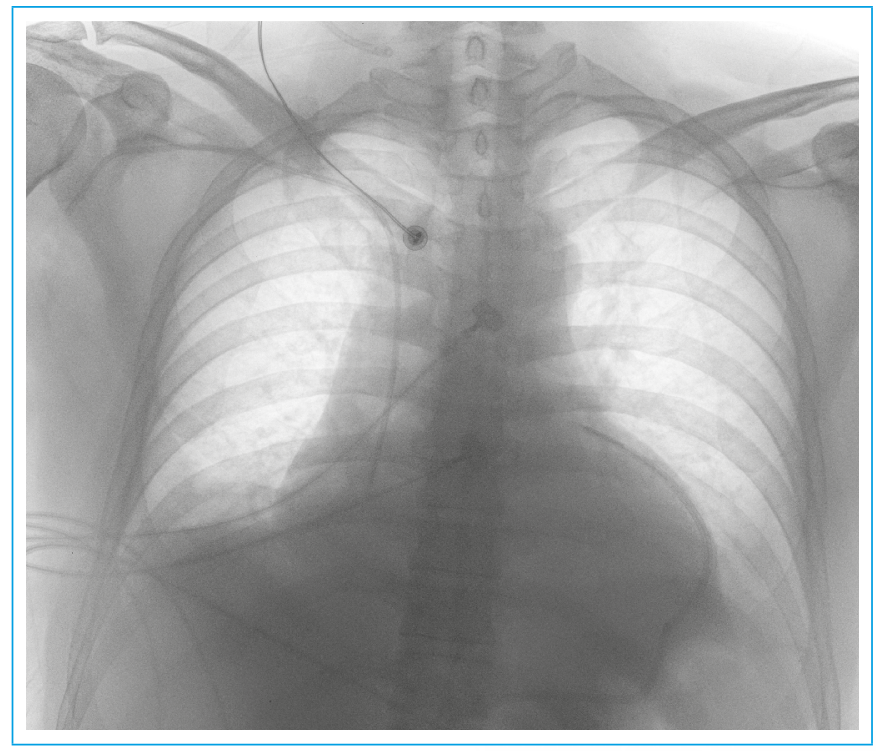

Figura 1. Radiografía de tórax, proyección PA. Se observa derrame pleural de predominio derecho y ensanchamiento de la silueta cardíaca

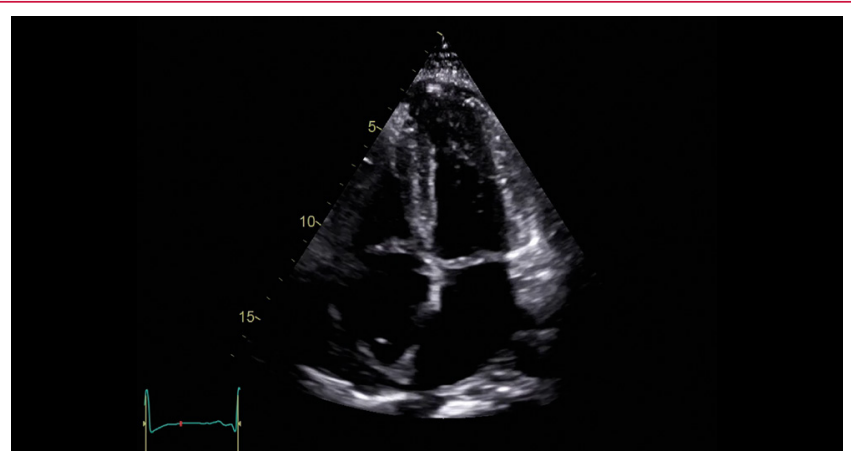

Vídeo 1. Ecocardiografía "normal" tras la pericardiocentesis. Plano apical de cuatro cámaras

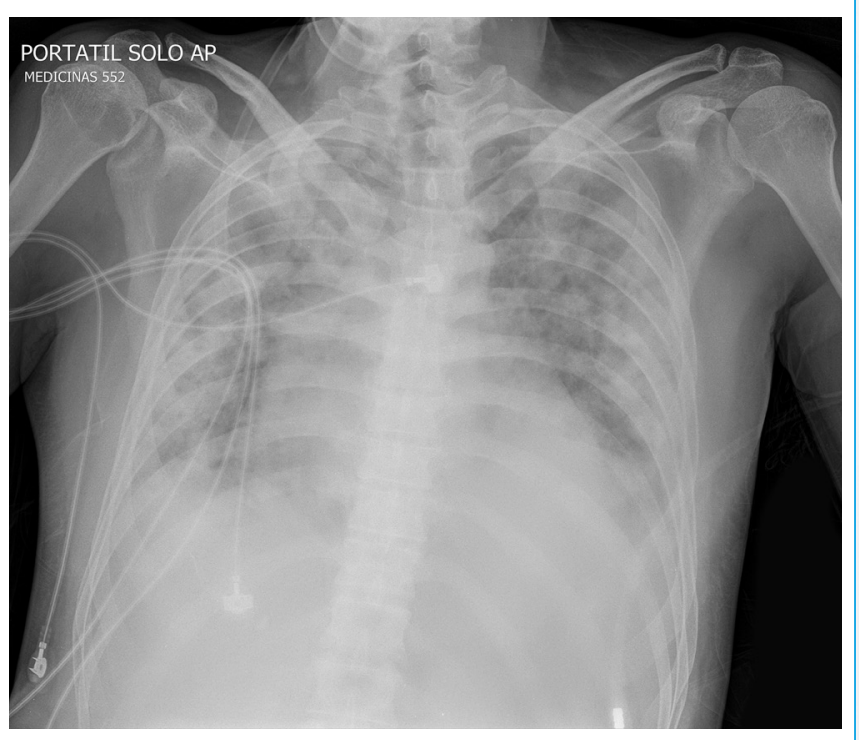

Figura 2. Radiografía de tórax. Se observa un infiltrado algodonoso en ambos campos pulmonares con derrame pleural de predominio izquierdo

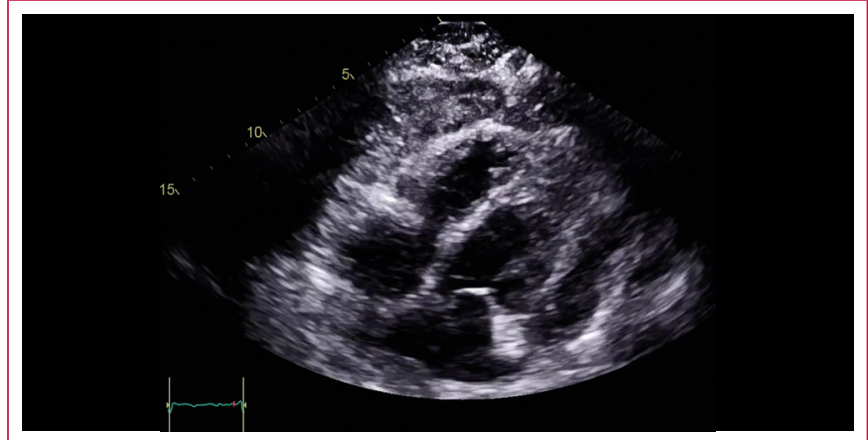

Vídeo 2. Ecocardiografía en el reingreso. Plano subcostal. Engrosamiento muy llamativo del pericardio. Movimiento paradójico del septo

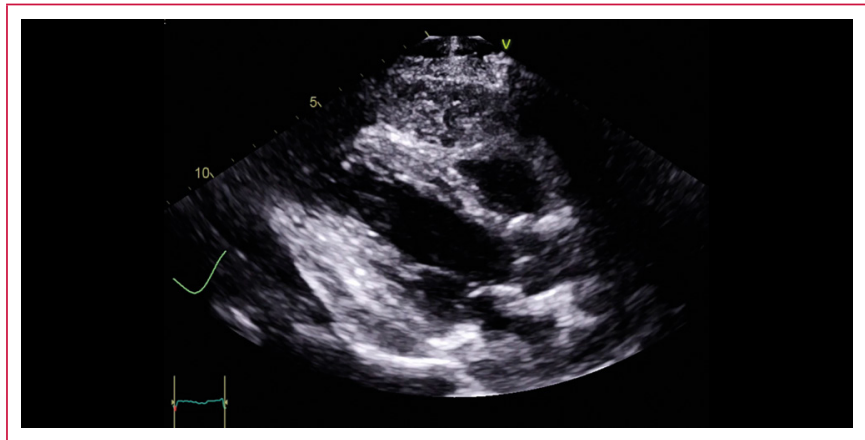

Vídeo 3. Ecocardiografía en el reingreso. Plano paraesternal. El pericardio aparece como una banda gruesa de tejido de densidad heterogénea

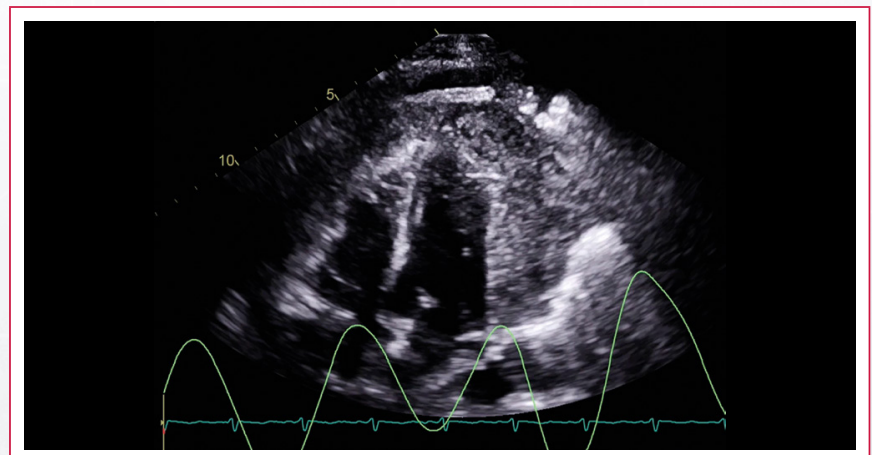

Vídeo 4. Ecocardiografía en el reingreso. Plano apical. Destaca de nuevo el terrible engrosamiento pericárdico. Además, se aprecia derrame pericárdico libre asociado

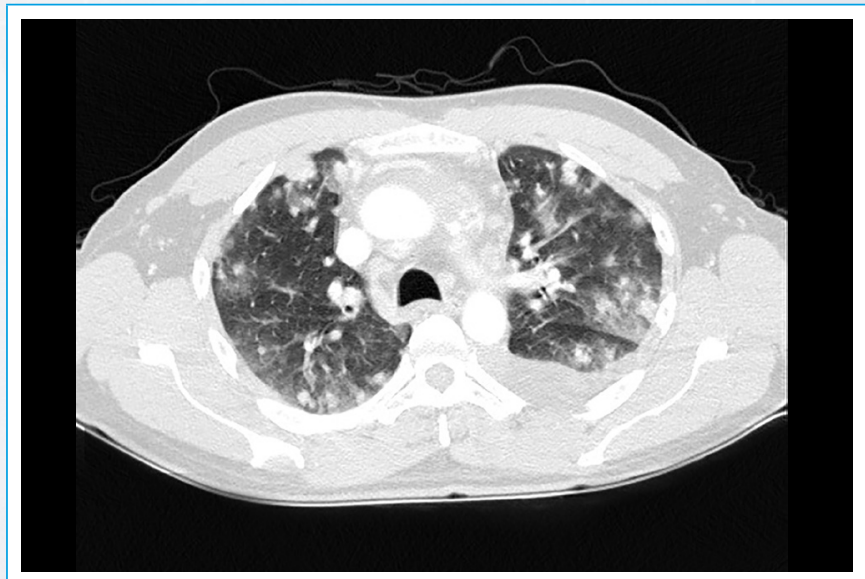

Figura 3. Corte axial de tomografía torácica con contraste. Se observan infiltrados nodulares bilaterales en vidrio deslustrado e imágenes sólidas asociadas a derrame pleural izquierdo 


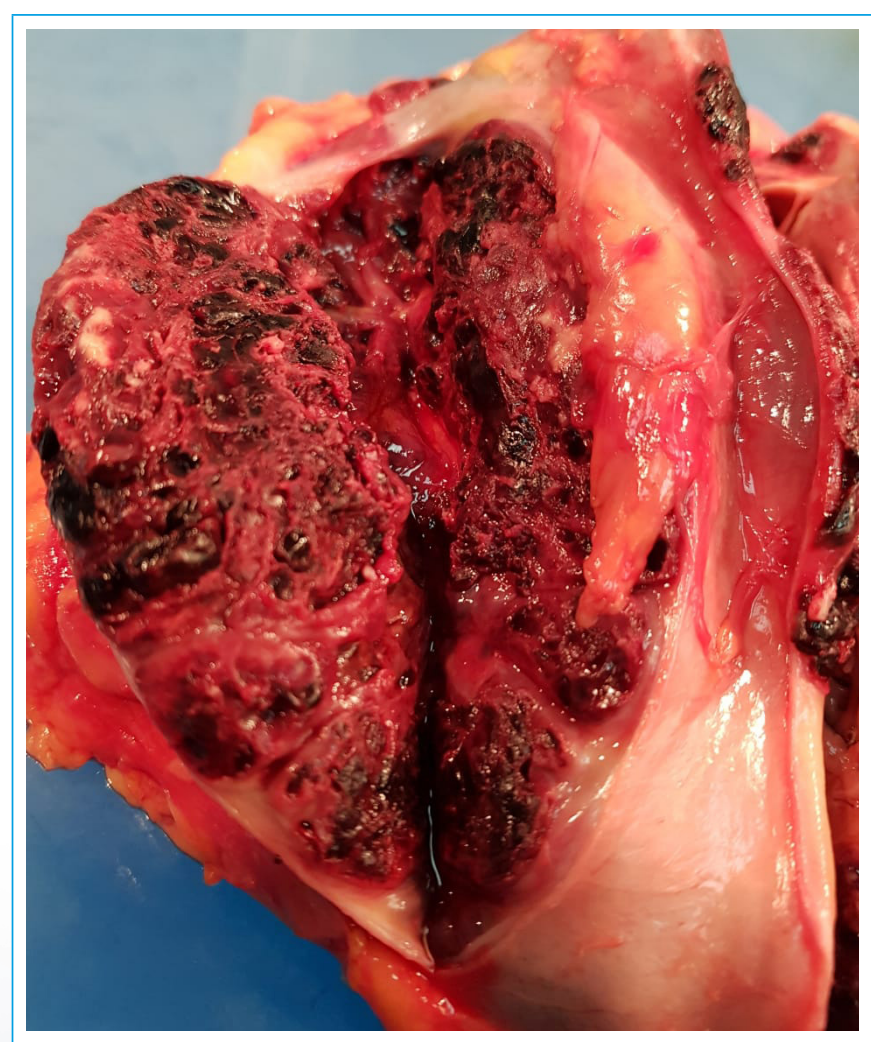

Figura 4. Pieza histológica del corazón donde se observan lesiones necrosadas infiltrantes en el pericardio formando granulomas (cortesía del Dr. Vargas, Servicio de Patología, Hospital San Vicente Paúl, Heredia, Costa Rica)

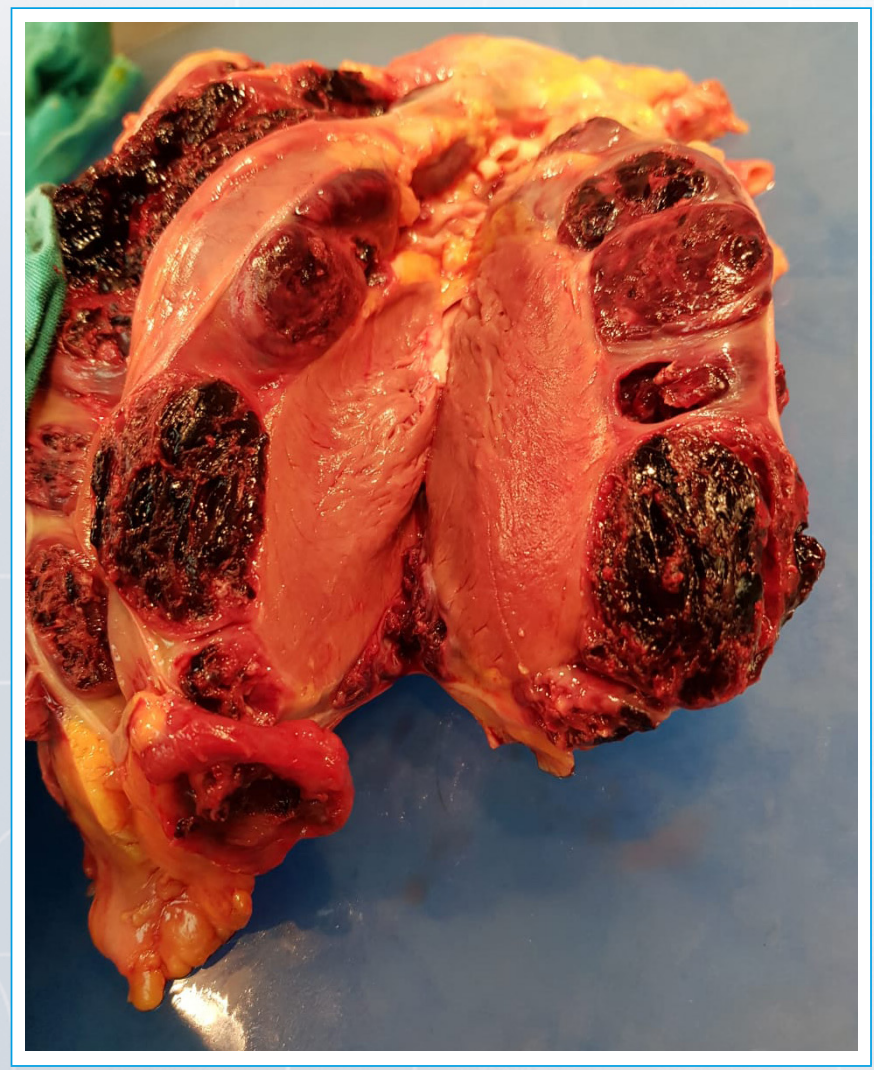

Figura 5. Otra vista de la pieza histológica de corazón donde se observan las lesiones infiltrantes en pericardio necrosadas formando granulomas (cortesía del Dr. Vargas, Servicio de Patología, Hospital San Vicente Paúl, Heredia, Costa Rica)

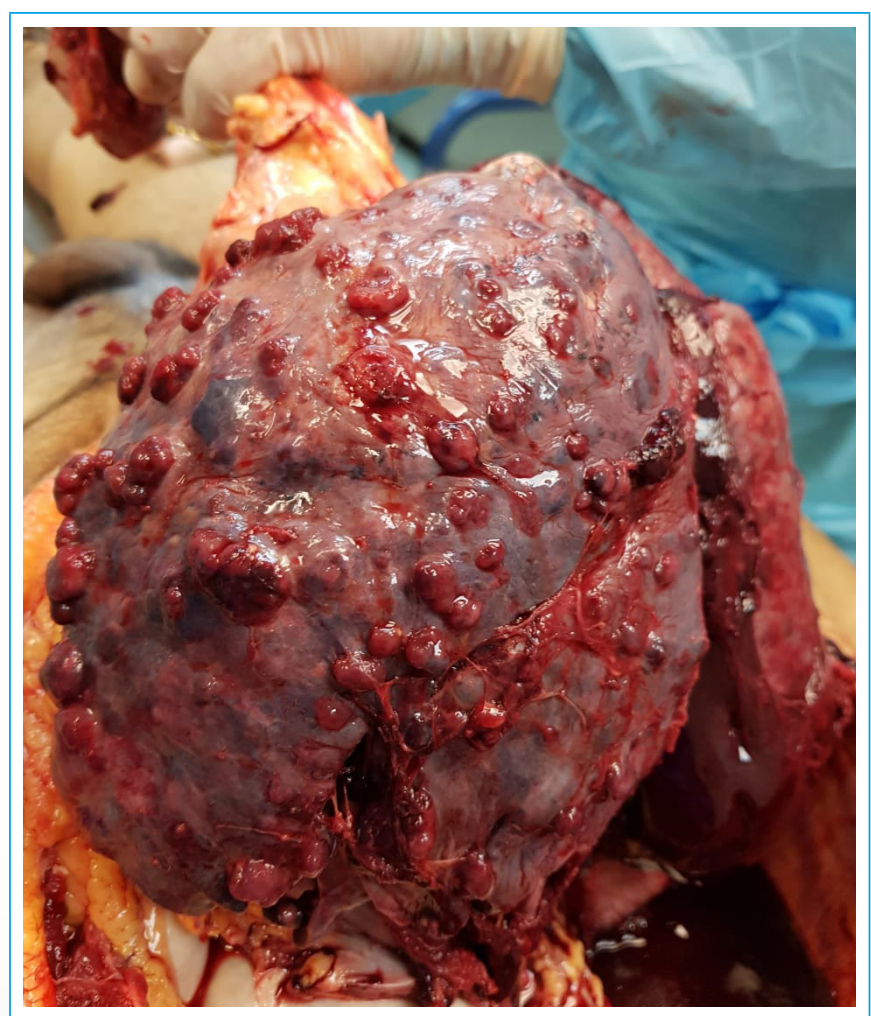

Figura 6. Pieza histológica del pulmón, con múltiples lesiones nodulares en el parénquima (cortesía del Dr. Vargas, Servicio de Patología, Hospital San Vicente Paúl, Heredia, Costa Rica)

\section{Discusión}

Los tumores cardíacos primarios son raros, con una incidencia entre el $0,0017 \%$ y el $0,03 \%$ de todos los tumores. De todos los tumores malignos cardíacos, los sarcomas son los más frecuentes $(76-78 \%)^{(1,2,3)}$ y el angiosarcoma representa aproximadamente el $31 \%$ de los tumores de estirpe maligna.

El angiosarcoma primario de pericardio es extremadamente raro y está asociado a muy mal pronóstico ${ }^{(4)}$. Con una mayor incidencia en varones, aparece entre la tercera y la cuarta década de la vida. Su progresión inicialmente es asintomática o muy inespecífica(5). Finalmente suele presentarse con insuficiencia cardíaca y es frecuente el derrame pericárdico severo.

En el paciente de este caso los diagnósticos diferenciales evaluados inicialmente fueron tuberculosis y miocarditis viral, pero las serologías virales, bacteriología y serologías para tuberculosis, fueron negativas. En la segunda internación fue imposible no pensar en una causa neoplásica por la rápida progresión de los síntomas y el deterioro marcado de su estado general. Llamó mucho la atención la rápida progresión de la enfermedad, sin poder tener un diagnóstico certero hasta después de la muerte del paciente. Apoyaba el diagnóstico de neoplasia maligna la infiltración pericárdica observada en la tomografía y la ecocardiografía, lo que hizo pensar en un proceso neoplásico infiltrativo del pericardio a pesar de no tener la confirmación histopatológica hasta el momento de su fallecimiento.

El angiosarcoma pericárdico se observa como lesiones rojas o pardas y hemorrágicas, mal circunscritas y que pueden invadir el miocardio subyacente, el pulmón o la pared torácica( ${ }^{(4)}$. En la autopsia las muestras macroscópicas revelaron características de tumor maligno con metástasis en el parénquima pulmonar. La histología con marcadores CD 31+ y CD 34 + llevó al diagnóstico final de angiosarcoma primario de pericardio. 
En estos pacientes se ha descrito una supervivencia promedio de 6 a 14 meses El tumor responde pobremente a la radioterapia y a la quimioterapia, y en muchos casos se recomienda un manejo conservador del paciente. Si se piensa en la cirugía, la resección del tumor(es) es compleja, ya que son masas muy friables con alto riesgo de hemorragia en el momento de las resecciones ${ }^{(5)}$.

\section{Conclusión}

El angiosarcoma primario de pericardio es un tumor extraordinariamente raro y de diagnóstico muy difícil. Tiene opciones terapéuticas muy limitadas y un pobre pronóstico(5).

\section{Ideas para recordar}

- Ante un derrame pericárdico hemorrágico se debe descartar la etiología maligna.
- La biopsia de pericardio se debe realizar tempranamente ante la sospecha de malignidad, a pesar de ser un procedimiento invasivo.

- Los tumores del pericardio son enfermedades raras que deben entrar dentro del diagnóstico diferencial.

\section{Bibliografía}

1. Randhawa J, Budd G, Randhawa M, et al. Primary cardiac sarcoma. American Journal of Clinical Oncology 2016; 39 (6): 593-599.

2. Yao H, Miyamoto T, Mukai S, et al. Angiosarcoma of the pericardium. The Japanese Journal of Thoracic and Cardiovascular Surgery 2003; 51 (2): 65-67.

3. Burazor I, Aviel-Ronen S, Imazio M, et al. Primary malignancies of the heart and pericardium. Clinical Cardiology 2014; 37 (9): 582-587.

4. Maleszewski J, Anavekar N. Neoplastic pericardial disease. Cardiology Clinics 2017; 35 (4): 589-600.

5. Herrmann M, Shankerman R, Edwards W, et al. Primary cardiac angiosarcoma: A clinicopathologic study of six cases. The Journal of Thoracic and Cardiovascular Surgery 1992; 103 (4): 655-664. 